\title{
Virtudes argumentativas: hacia una cultura de la paz
}

Mario Gensollen

Departamento de Filosofía

Universidad Autónoma de Aguascalientes

mgenso@correo.uaa.mx

\section{Argumentación y violencia}

Quisiera partir de un principio simple, el principio de la menor violencia:

(PMv) Frente a todo conflicto de creencias, sólo tenemos tres maneras de afrontarlo: mediante la violencia explícita, mediante la imposición externa, o mediante la argumentación. De las tres, la menos violenta es la argumentación.

Este principio supone, de entrada, que frente a un conflicto de creencias caben dos posibilidades iniciales: tratar de resolverlo o evitarlo ${ }^{1}$. Si lo tratamos de resolver, nuestras po-

1 No todo conflicto de creencias es afrontado, y no es conveniente afrontar todo conflicto de creencias. Además, saber cuándo es adecuado afrontar un conflicto de creencias es el primer paso de un ciclo argumental exitoso. Aristóteles recoge esta tesis, a modo de consejo práctico: «no hay que discutir con todo el mundo, ni hay que ejercitarse frente a un individuo cualquiera. Pues, frente a algunos, los argumentos se tornan necesariamente viciados: en efecto, contra el que intenta por todos los medios parecer que evita el encuentro, es justo intentar por todos los medios probar algo por razonamiento, pero no es elegante» (Top. 164b). 
sibilidades -aparentemente múltiples y variopintas- pueden reducirse, sin embargo, a las tres que PMv señala. Por un lado, podemos poner en marcha mecanismos externos y explícitos de violencia: gritos, golpes, guerras. También podemos dejar la solución a una tercera parte (externa ${ }^{2}$ ), un juez al que confiemos la (o se nos imponga su) decisión. Por último, podemos iniciar un ciclo argumental.

Las dos primeras formas de afrontar un conflicto de creencias son violentas de suyo. En el primer caso, la violencia es explícita y patente: deja la solución del conflicto a la fuerza (y sus múltiples formas, como el poder, el carácter o el miedo). En el segundo caso, la violencia se ejerce contra la voluntad de las partes: el perdedor tiene que aceptar su derrota, no a causa de su convencimiento, sino de la imposición de un tercero ${ }^{3}$.

Esto ya nos deja una idea vaga y preliminar de los mecanismos que inician el engranaje de nuestras argumentaciones:

a) Iniciamos ciclos argumentales cuando hay conflictos de creencias. Sin conflicto no hay argumentación, a lo más pedagogía argumentativa. Cuando acordamos, de inicio la argumentación se vuelve innecesaria. Los teólogos y los juristas debaten, cuando no hay conflicto, como un ejercicio pedagógico, como un mero entre-

\footnotetext{
2 Sabemos que desde la disputatio romana, se ha pensado la argumentación como constituida necesariamente por un proponente (proponens), un oponente (opponens o quaerens), y un juez (magister). Sin embargo, cabe señalar que el papel del juez puede ser o interno o externo a las partes del conflicto. A menudo cuando reflexionamos cumplimos nosotros mismos las tres partes, o bien cuando argumentamos con otra persona -no pocas veces- no hay un juez externo. La violencia de la que hablo surge cuando el juez es una tercera parte externa a las partes del conflicto.

3 Quizá sea ésta la razón del renovado interés por encontrar formas argumentativas -como la mediación y la negociación- que resuelvan conflictos sin necesidad de llegar al juicio. Para un análisis de estas formas argumentativas, ver Carnevale \& Pruitt 1992.
} 
namiento. La argumentación inicia a partir nuestro natural desacuerdo en muchas de nuestras creencias.

b) Argumentamos cuando deseamos resolver el conflicto evitando violencias externas: porque deseamos llegar, mediante el ejercicio de dar y recibir razones, a la forma menos violenta de acuerdo.

De estas dos anotaciones surgen algunas preguntas: ¿por qué los seres humanos tenemos desacuerdos de creencias?, ¿puede haber violencias internas a la argumentación?, ¿argumentar nos garantiza evitar cualquier forma de violencia?, ¿cuál es el fin o la meta de la argumentación?

Con respecto a la primera pregunta, los epistemólogos al día de hoy se cuestionan seriamente sobre la causa de nuestro natural (y habitual) desacuerdo. La llamada «epistemología del desacuerdo» trata de averiguar por qué los seres humanos no concordamos en muchas de las cosas que creemos (muchas de las cuales, además, nos importan). El problema no es simple: aunque no pocas veces el desacuerdo es fruto de la falta de información o razonamiento de alguna de las partes del conflicto, muchas veces el desacuerdo se da entre pares (personas igualmente informadas, racionales, virtuosas), o bien el conflicto tiene que ver con creencias donde no es nada sencillo saber (o determinar) quién tiene la razón.

La segunda y tercera preguntas ya suponen una posibilidad: que puede haber violencias internas a la argumentación. $Y$ es que argumentar no descarta la posibilidad de sucumbir ante la violencia. Muchas veces somos arrastrados por mecanismos que llevan a mal puerto la argumentación: simplificamos, complicamos, desviamos o detenemos los ciclos 
argumentales ${ }^{4}$. Las causas pueden ser varias: desde nuestro mal uso del lenguaje ${ }^{5}$ hasta nuestro deseo enloquecido por tener la razón (o evitar a toda costa tenerla). Argumentar no evita la violencia: se da a partir de la violencia misma del desacuerdo, y puede generar violencias que -si bien no son explícitas o externas a la argumentación- extravíen nuestros ciclos argumentales. A pesar de todo, argumentar es la forma menos violenta de resolver conflictos de creencias.

La última pregunta, por su parte, genera perplejidad: la tradición ha visto como la meta de la argumentación la victoria. Ha planteado el escenario en términos bélicos: cuando argumentamos satisfactoriamente -se ha creído- siempre habrá un vencedor y un vencido. Siempre, alguna de las partes tendrá que rendirse ante mejores razones que las suyas. Esta forma de comprender la argumentación es atractiva y no deja de ser interesante: supone -acertadamente- que siempre habrá mejores razones que otras, que frente a todo argumento siempre habrá un mejor contraargumento. Sin embargo, dicha forma de entender la argumentación deforma algunas de nuestras prácticas argumentativas, y no se percata de que muchas de las creencias en conflicto cuando argumentamos no son creencias entre las que sea posible decantarse de manera contundente.

Tenemos que atender entonces a la variedad inmensa de nuestras prácticas argumentativas. No siempre que argumentamos buscamos lo mismo: la variedad de nuestros in-

\footnotetext{
A estos mecanismos, Pereda (ver 1994a; 94-133) los llama «vértigos argumentales», y hace una caracterización exhaustiva de los mismos.

5 La falta de claridad detiene constantemente la argumentación, haciendo necesario que las partes del conflicto tengan que ir atrás y ejecutar ciclos reconstructivos, donde la meta será «hablar mejor de lo mismo», para reanudar posteriormente el ciclo argumental. Ver Pereda 1994b.
} 
tereses tanto teóricos como prácticos cuando argumentamos merecería un examen detenido. Algunas veces buscamos la verdad, otras el acuerdo respetando en parte el desacuerdo, otras la simple solución de un conflicto práctico. En ciencia, el esquema tradicional parecería funcionar casi a la perfección: desechamos a veces teorías como falsas, otras veces nos decantamos ante alguna como la más cercana a la verdad. Pero el punto es que en dichas prácticas argumentativas es la verdad lo que está en juego: e.g., es falso que el arsénico cura el dolor de cabeza, así como es verdadero que nuestro planeta gira en una órbita elíptica alrededor del Sol. Lo que está en juego no son normas, sino descripciones del mundo (no obstante, es muy debatible si podemos excluir por completo la normatividad en nuestras descripciones del mundo). Incluso las prácticas científicas son numerosas, diversas y complejas: no es una obviedad para qué se argumenta en cada uno de los casos.

Otras veces no se busca la verdad, sino el acuerdo: para ello el esquema tradicional fracasa rotundamente. Lo que se busca en ocasiones es llegar a un acuerdo particular a pesar de que las diferencias persistan en otros aspectos. Esto sucede de manera paradigmática en argumentaciones donde las creencias en disputa tienen un carácter normativo: donde entran en escena valores, gustos, preferencias, deseos, expectativas, etcétera. En estos casos resulta de lo más complejo esperar que en nuestros ciclos argumentales haya vencedores y vencidos: este esquema fracasa pues las partes irán casa con el sentimiento de no haber sido comprendidos, o con el sentimiento (incluso más feroz) de no haber comprendido al otro. Las metas de nuestras argumentaciones, por tanto, son de lo más diversas: el error, muchas veces, consiste en ni siquiera ser conscientes de para qué estamos argumentando, o qué buscamos mientras lo hacemos. 
Hasta este momento algo he dicho de la constitución de nuestras argumentaciones: por qué empezamos a argumentar, y qué buscamos cuando argumentamos. Sin embargo, poco o nada he dicho de su normatividad: qué deberíamos hacer para llevar a buen puerto el ejercicio de dar y recibir razones. La tradición también tiene una respuesta que no me parece satisfactoria del todo: se ha concentrado en los argumentos y no en quién argumenta, ni en las circunstancias en las que lo hace. Se ha estudiado con detenimiento qué hace que nuestras razones sean satisfactorias o insatisfactorias, y esto con independencia del contexto en el que se usan y de quién las usa.

\section{La argumentación situada}

Partamos de otro principio, el principio de la argumentación situada:

(PAS) Toda argumentación está situada: se da en un terreno común, presupone cosas cuando inicia y termina presumiendo cosas.

Este principio asume que todo ciclo argumental siempre se da en medio: parte de un terreno común que comparten las partes en conflicto (presupone un lenguaje común, creencias, intereses, etc.), y -si llega a buen puerto- termina presumiendo al menos algunos contenidos proposicionales que es posible inferir a partir de los contenidos proposicionales a los que se ha llegado por medio de la argumentación.

Empecemos por el terreno común. Para siquiera poder iniciar un ciclo argumental, una condición necesaria es que las partes en conflicto compartan un sinnúmero de creencias 
(entre otras muchas cosas). Las creencias en disputa se dan sobre una sólida y vasta meseta de creencias compartidas. Sin ellas, no sería posible evaluar otras creencias, ni las razones a favor o en contra de ellas. Estas presuposiciones ${ }^{6}$ funcionan de manera peculiar. Cuando hablamos presuponemos ciertas cosas, y lo que presuponemos guía tanto lo que decidimos decir, como el cómo intentamos que sea interpretado lo que hemos dicho. La presuposición de un hablante es una actitud proposicional: presuponer algo es darlo por sentado, o al menos actuar como si uno lo diera por sentado, como información de fondo -como terreno común entre los participantes de la conversación-. Lo que es más distintivo de esta actitud proposicional es que es una actitud social o pública: alguien presupone que $\phi$ sólo si presupone que otros lo presuponen de la misma manera (ver Stalnaker 2002; 701).

En este sentido, el terreno común se constituye de un conjunto de creencias que un hablante presupone, dándolas por sentadas, o al menos actuando como si las diera por sentadas. La dimensión social de la presuposición indica algo más: uno presupone sólo si presupone que los otros lo presuponen. Esto muestra que puede haber errores de presuposición. Ellos, al menos de inicio, pueden ser los candidatos para explicar ciertos bloqueos argumentativos.

Ahora bien, si todo ciclo argumental inicia desde cierto o ciertos puntos de vista -está situado- y toda argumentación

\footnotetext{
6 Contra la tradicional caracterización de Frege y Russell, atiendo a un sentido pragmático de «presuposición», como condiciones de afirmabilidad de expresiones o como requisitos para la ejecución de actos de habla en un determinado contexto. Ver Strawson 1964, Stalnaker 1974, y Gazdar 1979. Algunos pragmatistas analizan las presuposiciones a partir del principio griceano de racionalidad comunicativa y las máximas de conversación que se derivan de éste (ver Grice 1975): así, las presuposiciones se identificarían con las implicaciones pragmáticas -implicaturas convencionales y conversacionales (ver Grice 1981). Para un recuento histórico de estas posiciones, ver de Bustos 2011.
} 
siempre está en medio tanto de ciertas presuposiciones, no se puede empezar a argumentar de cero, y toda argumentación deja siempre cabos sueltos. El terreno común entre las partes es el que sitúa al ciclo argumental: sin este situarse inicial el ciclo no podría comenzar. Sin embargo, los bloqueos pueden ser posteriores a su inicio; pueden darse cuando el ciclo argumental ya está en funcionamiento.

En algún sentido, ignorar o desatender el terreno común (cometer errores de presuposición) genera bloqueos argumentativos: las partes comúnmente requieren ir atrás del punto que se discute. Esto pasa con mucha frecuencia: $A$ argumenta en favor de $p$, pero su argumento en favor de $p$ presupone $q ; B$, por el contrario, argumenta en favor de $-p, y$ su argumento presupone que $-q$. El ciclo argumental se detendrá, y dará un paso atrás: ahora es necesario argumentar en favor de $q$ o en su contra. Otra forma de hacer frente a un conflicto como el anterior, y quizá la forma más razonable de abordarlo, es argumentar sin presuponer la verdad o la falsedad de $q$, esgrimiendo argumentos que no presupongan ni $q$ ni $-q$. En este caso, $q$ no puede formar parte del terreno común sin generar bloqueos argumentativos.

Toda argumentación, además, termina presumiendo algunas cosas. De entrada, por «presunciones» entiendo «expectativas razonables con respecto al futuro». En el terreno jurídico, sabemos que "cualquier acusado de un crimen es inocente hasta que se demuestre lo contrario». Así, en casos como el anterior, las presunciones operan como meta-reglas de inferencia: se trata, en el ejemplo citado, de satisfacer la falta de información a la que se enfrenta un juez. En los textos de jurisprudencia, las presunciones funcionan como 
«consecuencias que un juez extrae de un hecho conocido a un hecho desconocido» ${ }^{7}$.

En la vida cotidiana, las presunciones dirigen nuestra atención hacia el futuro, produciendo expectativas razonables. Toda argumentación, cuando termina, presume algunas consecuencias de lo acordado. Presume algunos contenidos proposicionales que es posible inferir (a veces gradualmente) de los contenidos proposicionales en los que se expresa el acuerdo: por ello, es posible evaluar argumentos no sólo por lo que expresan explícitamente, sino por alguna o varias de sus consecuencias. También, toda argumentación presume alguna o algunas acciones que se siguen del acuerdo: e.g., actuar como si $p$ fuese verdadero, deseable, correcto, adecuado (siendo $p$ el contenido proposicional del acuerdo al que se ha llegado).

Así, toda argumentación está situada: inicia necesariamente con presuposiciones acerca de creencias, deseos, intereses de las partes en conflicto; y termina presumiendo contenidos proposicionales y acciones que se siguen de los acuerdos a los que han llegado las partes.

\section{Virtudes argumentativas}

A partir del PMV y del PAS, ahora podemos atender a otro principio, el principio de las virtudes argumentativas:

Para una caracterización de las presunciones como reglas procedimentales constitutivas de nuestros ciclos argumentales; ver Pereda 1994a, 51-5. Sin embargo, deformo bastante la caracterización de Pereda, pues éste parece unir las presunciones a las presuposiciones bajo el mismo término. 
(PVA) Para alcanzar las metas de nuestros ciclos argumentales, no basta con atender a los argumentos y a su contexto, sino a quienes argumentan.

Anteriormente atendí al contexto común de las partes que tratan de resolver un conflicto de creencias dando y recibiendo razones. Las argumentaciones se sitúan en dicho contexto, el cual está determinado por las presuposiciones y las presunciones del ciclo argumental. No pocas veces argumentamos mal, o no logramos la meta de la argumentación, por desatender a dicho contexto. Sin embargo, además del contexto, el pVA nos dice que debemos atender a los agentes de las prácticas argumentativas. Para ello, debemos enfocar nuestra atención en algunos de sus rasgos de carácter, los cuales pueden favorecer o truncar el posible acuerdo que se busca cuando argumentan.

Dicho de manera opaca: para alcanzar las metas de nuestras argumentaciones debemos saber argumentar, atender al contexto en el cual estamos argumentando y ser virtuosos argumentativamente. Diré algo de cada uno de estos requerimientos.

Como he dicho, la tradición ha enfocado su atención en los argumentos, con independencia del contexto de la argumentación y de los sujetos que argumentan. Así, tradicionalmente se entiende por «argumento» una secuencia de oraciones tal que las premisas están al comienzo y la conclusión al final. Ha sido principalmente la lógica formal la encargada de estudiar los vínculos entre las premisas y la conclusión de los argumentos -donde conceptos como «consecuencia lógica» y «validez» son claves-, entendidos de esta forma restrictiva. Así, una persona que desea argumentar adecuadamente-nos diría el lógico formal- debería ser capaz de reconocer la cuan- 
tificación de una frase, las funciones de verdad y -a partir de éstas- poder reconocer tautologías, contradicciones, contingencias proposicionales y contraejemplos. Sin embargo, el conocimiento de la lógica formal no basta para nuestras argumentaciones cotidianas, pues para ellas se requiere -entre otras cosas- conocimiento, disposición y habilidad para: organizar una discusión, apoyar con razones, aceptar consecuencias, saber cuándo y qué preguntar, clarificar el discurso, reconocer la estructura del argumento, saber cuándo la evidencia es suficiente e insuficiente, y buscar alternativas ${ }^{8}$.

Ahora, ¿qué se requiere para atender al contexto? El agente argumentativo debe ser capaz de reconocer las creencias presupuestas en la argumentación, y qué creencias es innecesario, o hasta improductivo, presuponer si se desea resolver el conflicto. También debe ser capaz de vislumbrar las presunciones que se siguen de lo acordado, y a qué acciones está comprometido si acepta los términos del acuerdo.

Por último, ¿qué rasgos de carácter es necesario que posea quien se dispone a resolver un conflicto argumentando? Quien argumenta requiere de ciertas actitudes virtuosas para llegar a las metas de sus prácticas argumentativas. Un examen detenido de cada una de estas virtudes excede los límites de este texto, pero mencionaré y describiré brevemente cinco que pienso como básicas:

a) Buena voluntad: quien no está dispuesto no sólo a afrontar argumentativamente un conflicto de creencias, sino a querer hacerse entender y entender a su interlocutor, así como a aceptar las consecuencias de

\footnotetext{
Un muy interesante recuento de lo que debe saber una persona educada para argumentar lo hace Morado (2009; 9-16).
} 
la discusión argumentada, es un mal agente argumentativo. Aristóteles llamó a esta disposición eunoia (ver Rhet. II, 1, 1377b22-24).

b) Caridad: muy cercana a la buena voluntad, la caridad implica que el agente argumentativo suponga máxima racionalidad y coherencia en su interlocutor. $\mathrm{Mu}-$ chas veces el error consiste en suponer que nuestro interlocutor es irracional (externamente): somos virtuosos, por el contrario, cuando incluso estamos dispuestos a reconstruir de la mejor manera las razones de nuestro opositor.

c) Falibilismo: aunque el falibilismo como posición teórica es cuestionable (ver Hurtado 2000), como actitud es virtuosa. La virtud argumentativa del falibilismo supone que el agente argumenta asumiendo que cualquiera de sus creencias que están en disputa puede ser falsa.

d) Coalescencia: el agente argumentativo es coalescente cuando da prioridad al acuerdo y a la solución del conflicto, o a la verdad, que a su victoria en la disputa ${ }^{9}$.

e) Cosmopolitismo y pluralismo: como dos caras de la misma moneda, el cosmopolita y pluralista sabe que las personas somos distintas, y podemos aprender mucho de nuestras diferencias. Éste ni desea ni espera que todas las personas y sociedades converjan en un único modo de vida. Sabe que cualesquiera que sean nuestras obligaciones con los otros (o las de los otros con nosotros), a menudo ellos tienen derecho de creer y hacer las cosas a su modo ${ }^{10}$.

Extraigo esta noción de Gilbert (2009).

10 Esta noción, que agrupa cosmopolitismo y pluralismo, Appiah (2006) la llama «cosmo- 


\section{Hacia una cultura argumentativa}

Terminaré con una serie de insinuaciones, más que con una teoría delineada en todos sus puntos. Para ello, quisiera atender a un último principio, el principio de la cultura argumentativa:

(PCA) La forma más adecuada para erradicar la cultura de la violencia es la cultura argumentativa.

Inicié sugiriendo, a partir del PMV, que argumentar es la forma menos violenta de resolución de conflictos de creencias. De entrada, el conflicto es violento: los seres humanos creemos cosas distintas, y es inevitable que este hecho genere algunas ocasiones diversos problemas. Disponemos de formas más o menos eficaces de resolver los conflictos: podemos operar con violencia explícita, o tratar de resolver el conflicto dando y recibiendo razones. He dicho también que argumentar no necesariamente evita la violencia: podemos fallar argumentativamente y generar violencia posterior a nuestra argumentación, o bien podemos sucumbir ante violencias internas a nuestro ejercicio de dar y recibir razones. Para ello, sugerí que es necesario atender a los argumentos, pero también al contexto, y a los rasgos del carácter deseables que debe poseer el que argumenta (a las virtudes deseables para llevar a buen puerto los ciclos argumentales). El problema, como puede verse, es la violencia, y el supuesto de que deseamos evitarla en la medida de lo posible.

Todos sabemos que hay contextos sociales que favorecen la violencia. Asimismo, otros la moderan. Un entorno social

$\overline{\text { politismo parcial». Para una defensa de un pluralismo no relativista, ver Pettit } 2000 .}$ 
en el que existen -entre otras cosas- sistemas legales, y valores cívicos y morales lo suficientemente sólidos, modera nuestra natural tendencia a la agresividad. Por tanto, la violencia es más común y sus índices son más altos en sociedades donde la legalidad es débil, en sociedades corruptas, en sociedades donde priman la pobreza y la excesiva desigualdad. Estos factores contextuales son los que en vez de moderar las tendencias y emociones que nos inducen a la agresividad y la violencia, hacen que deriven en patrones violentos de conducta que terminan por destruir el tejido social.

Se sabe ya -aunque algunos aún lo discutan- que la moralidad tiene una base biológica compuesta principalmente por tendencias y emociones. Ahora bien, dado que éstas tienen una orientación práctica, es indispensable construir -o reconstruir- un entorno adecuado para que éstas puedan desplegarse de un modo más armónico. Los entornos violentos generan más violencia. Como lo han señalado recientemente las neurociencias, ante el exceso de violencia nuestro cerebro inhibe determinadas emociones y exacerba otras (e.g., el miedo). De ahí que podemos decir que, aunque naturalmente parecemos rechazar la violencia, en cierto modo podemos acostumbrarnos a ella ${ }^{11}$.

Cuando el clima de la violencia se ha expandido y se ha vuelto moneda común en la sociedad, la sanación social sólo puede ser paulatina. La violencia no es -como pudiera pensarse- exclusivamente un problema de Estado. Es, ante todo, un problema humano. El fortalecimiento de una cultura de la paz podría resultar un remedio viable. Pero, como sugerí en el PCA, se trata de replantear, de reconstruir el entorno

11 Uno de los análisis más lúcidos al respecto lo brindó Susan Sontag (2004) a raíz de las torturas cometidas por soldados norteamericanos a presos iraquíes en la prisión de Abu Ghraib. 
social que modere y module nuestras tendencias biológicas que pueden conducirnos a la violencia.

La reconstrucción de la solidaridad, la fraternidad, la empatía y el reconocimiento entre unos y otros, son valores necesarios cuando se aspira a una verdadera cultura de la paz (sólo dentro de la cual es viable una cultura democrática).

La agresividad y la violencia operan como formas de resolución de conflictos. Pero no todo conflicto es del mismo tipo $\mathrm{y}$, por tanto, no todas las formas de resolución de conflictos deben ser las mismas. Una cultura de la paz y una cultura democrática requieren al menos de dos mediaciones si es deseable evitar las distintas formas de violencia: la argumentación y la legalidad. Los humanos argumentamos cuando deseamos resolver un conflicto, pero el correcto argumentar tiene requisitos indispensables - de los cuales ya he hablado-, y los cuales en su conjunto pueden llegar a constituir una cultura argumentativa. Pero no basta. También se requieren instituciones robustas y confiables en el terreno legal: cuando es imposible argumentar, ya sea por falta de interés entre las partes o por carencia de una cultura argumentativa, se necesita de un tercero virtuoso que resuelva el conflicto.

Esta reconstrucción exige una labor transdisciplinaria. La cultura, la educación, la ética, pueden reflorecer aun cuando el deterioro social sea grave. Es la sociedad civil, no sólo el Estado, la encargada de promover, construir y fortalecer el clima propicio para contrarrestar la violencia. No se trata únicamente de replantear las políticas públicas sino de generar conjuntamente una nueva cultura. El primer paso puede ser la promoción de una cultura argumentativa ${ }^{12}$.

12 Debo muchas de estas últimas ideas a Luis Xavier López-Farjeat (ver 2011), y a nuestras numerosas discusiones a este respecto. A él, un sincero agradecimiento. 


\section{BIBLIOGRAFÍA}

Appiah, K. A. (2006). Cosmopolitanism: Ethics in a World of Strangers. New York: W. W. Norton \& Company. Aristóteles. (1994a). Tratados de lógica I. Madrid: Gredos. . (1994b). Retórica. Tr. Quintín Racionero. Madrid: Gredos.

Baghramian, M. \& Ingram, A. (eds). Pluralism: The Philosophy and Politics of Diversity. New York: Routledge.

Carnevale, P. J. \& Pruitt, D. G. (1992). «Negotiation and Mediation». Annu. Rev. Psychol. 43; 531-82.

Cole, P. (ed). (1981). Radical pragmatics. New York: Academic Press.

de Bustos, E. (2011). «Presuposición». En Vega \& Olmos (2011); 483-5.

Gazdar, G. (1979). Pragmatics: Implicature, presupposition and logical form. New York: Academic Press.

Gilbert, M. (2009). Coalescent argumentation. New York: Routledge.

Grice, P. (1981). «Presupposition and conversational implicature». En Cole (1981); 183-98.

. (1975). «Logic and conversation». En Study in the way of words (pp. 22-40). Cambridge, MA: Harvard University Press, 1989.

Hurtado, G. (2000). «Por qué no soy falibilista». Crítica 32 (96); 59-97.

López-Farjeat, L. X. (2011). «La naturaleza de la violencia». Conspiratio 12; 44-53.

Morado, R. (2009). Compendio de lógica. México: Editorial Torres Asociados.

Munitz, M. K. \& Unger, P. (eds). (1974). Semantics and Philosophy. New York: New York UP. 
Pettit, Ph. (2000). «A Sensible Perspectivism». En Baghramian \& Ingram (2000); 60-82.

Pereda, Carlos. (1994a). Vértigos argumentales. Una ética de la disputa. Barcelona: Anthropos-UAM.

. (1994b). «Hablar mejor de lo mismo». Diánoia XL; 53-83.

Sontag, Susan. (2004). Regarding the pain of others. New York: Picador.

Stalnaker, R. (2002). «Common Ground». Linguistics and Philosophy 25 (5-6); 701-21. . (1974). «Pragmatic presuppositions». En Munitz \& Unger (1974); 197-213.

Strawson, P. F. (1964). «Intention and Convention in Speech Acts». Philosophical Review 73; 439-46.

Vega Reñón, L. \& Olmos Gómez, P. (eds). (2011). Compendio de lógica, argumentación y retórica. Madrid: Trotta. 


\section{RESUMEN}

En este artículo se defiende que la promoción de una cultura argumentativa es el mejor camino para lograr una cultura de la paz. Para ello, se afirma que la argumentación es la forma menos violenta de resolver conflictos de creencias; y se sugiere que debemos tomar en cuenta tanto la estructura lógica de los argumentos, el contexto en el que son proferidos, así como algunos rasgos de carácter del agente o virtudes argumentativas.

Palabras clave: argumentación; violencia; virtud; presuposición; presunción.

\section{ABSTRACT}

In this paper I argue that the promotion of an argumentative culture is the best way to achieve a culture of peace. To do this, argumentation is the least violent way to resolve conflicts of beliefs; we should take into account the logical structure of the arguments, the context in which they are uttered, and some argumentative virtues of the agent.

Key words: argument; violence; virtue; presupposition; presumption. 